\title{
Strategies for in vivo genome editing in non-dividing cells
}

Fatemeharefeh Nami ${ }^{1}$, Mohsen Basiri ${ }^{2}$, Leila Satarian' ${ }^{2}$ Hossein Baharvand ${ }^{2,3 *}$, Catherine Verfaillie ${ }^{1 *}$

${ }^{1}$ Department of Development and Regeneration, KU Leuven-Stamcelinstituut, Leuven, Belgium

2 Department of Stem Cells and Developmental Biology, Cell Science Research Center, Royan Institute for Stem Cell Biology and Technology, ACECR, Tehran, Iran

3. Department of Developmental Biology, University of Science and Culture, Tehran, Iran

*Correspondence:

catherine.verfaillie@kuleuven.be (C. Verfaillie)

baharvand@royaninstitute.org (H. Baharvand)

Key words: gene editing, non-dividing cells, in vivo, homology directed repair, nonhomologous end joining.

\section{Abstract}

Programmable nucleases, including Zinc Finger Nucleases (ZFNs), Transcription Activator-Like Effector Nucleases (TALENs), and Clustered Regularly Interspaced Short Palindromic Repeats (CRISPR) have enhanced our ability to edit the genome by sequence specific generation of a double strand break (DSB) with subsequent homology directed repair (HDR) of the DSB. However, the efficiency of the HDR pathway is limited in non-dividing cells, which encompass the majority of in the body. Therefore, the HDRmediated genome editing approach has, reportedly, limited in vivo applicability. Here we discuss a mutation-type oriented viewpoint of strategies devised over the last years to circumvent this problem, their possible applications and limitations. 


\section{Glossary}

Base editors (BE): a fusion protein consisting of a cytidine deaminase and a dCas9/Cas9 nickase, capable of performing a C to T base conversion.

Clustered regularly interspaced short palindromic repeats (CRISPR): a defensive tool of archea and bacteria which has been harnessed as a programmable nuclease for genome editing in animals and plants.

Dead Cas9 (dCas9): a catalytically inactive form of cas9 generated by two silencing mutations in RuvC and HNH catalytic domains of Cas9 (D10A and H840A).

Homology directed repair (HDR): One of the two main DSB repair pathways which uses a template to restore the lost sequence at DSB site. Repair template can be a sister chromatid, other chromosome or duplicated sequence on the same or other chromosomes as well as exogenously provided templates.

Homology-independent targeted integration (HITI): a gene editing knock in strategy with a special design based on NHEJ ligation of a donor DNA mainly in a correct orientation.

Homology-mediated end joining (HMEJ): a gene editing strategy which uses a donor with homology arms similar to HDR donors but the donor is excised from the backbone inside the cell.

Microhomology-mediated end joining (MMEJ): an end joining repair pathway works independent of $\mathrm{Ku}$ and relies on microhomologous sequences of 5-25 bp size for sticking the broken ends.

Mismatch repair (MMR): a major evolutionary conserved DNA repair pathway which corrects base-base mismatches and insertion deletion loop of newly synthetized DNA.

Non-homologous end-joining (NHEJ): One of the two main DSB repair pathways which is donor-independent and uses heterodimeric enzyme consist of proteins Ku70 and Ku80, the catalytic subunit of protein kinase and ligase IV/XRCC4.

Obligate ligation-gated recombination (ObLiGaRe): a gene editing knock in strategy resembling $\mathrm{HITI}$, which uses obligated heterodimeric ZFN or TALEN. 
Precise integration into target chromosome (PITCH): a gene editing knock in strategy based on MMEJ pathway.

Protospacer adjacent motif (PAM): a conserved motif which might reside in $5^{\prime}$ or $3^{\prime}$ location relative to protospacer and is used by CRISPR system to determine the invader from endogenous target sequences.

Recombinase Cas9 (recCas9): a chimeric protein made of a serine recombinase and a cas9.

Transcription coupled NER (TC-NER): a subtype of nucleotide excision repair pathway which removes lesions from template DNA in actively transcribed genes.

Transcription activator-like effector nuclease (TALEN): a programmable nuclease made by fusion of TALE protein and Fokl cleavage domain. TALEN consist of a series of 33 to 35 amino acids repeats and a Fokl cleavage domain. Dimerization of Fokl monomers from two arms of TALEN results in DSB formation.

Zinc finger nuclease (ZFN): a programmable nuclease made by fusion of zinc finger proteins and a Fokl cleavage domain. DSB forms upon Fokl dimerization of two zinc finger proteins. 


\section{A case for novel in vivo gene editing strategies}

There are around 5000 monogenic disorders in humans wherein the gene mutation causes impaired function of terminally differentiated tissue cells (www.omim.org/statistics/geneMap). Although rare individually, collectively, monogenic disorders constitute a common health problem (http://www.orpha.net). A number of therapies exist for some monogenic of these disorders, including among others, organ transplantation or cell therapy, gene augmentation, RNA or protein therapies or metabolic manipulation. However, many monogenic disorders remain untreatable [5-9]. As the basic cause of these diseases is a mutated gene, gene editing would theoretically be able to resolve all of these disorders. In vivo genome editing has the advantage that gene expression remains under the control of the endogenous gene regulatory elements; and that the therapy is permanent [1]. However, to reverse the genetic defects in terminally differentiated cells, therapies that can correct the genetic defect in non-dividing cells affected by the monogenic disease is required, which remains a major challenge.

Genome editing using programmable nucleases such as ZFNs (see Glossary), TALENs, and CRISPR/Cas is based on generating DNA double strand breaks (DSB) in the sequence to be edited (Box 1) [10]. Such a DSB is sensed by cellular repair pathways, both nonhomologous end-joining (NHEJ) or homology directed repair (HDR). While NHEJ is generally known to be an error prone pathway causing insertions and deletions (indels), which occurs independent of a donor template, HDR is donor-dependent and is considered to be an error-free pathway. Therefore, HDR has been extensively tested and used for precise gene editing. As HDR is restricted to the $S$ and $G 2$ phases of the cell cycle, this approach is not suited for editing and repairing the genome in non-dividing cells with the exception of some specific circumstances (Box 2) [11]. Recently a number of novel genome-editing strategies [e.g. obligate ligation-gated recombination (ObLiGaRe), homology-independent targeted integration (HITI), Cpf1, precise integration into target chromosome (PITCH), homology-mediated end joining (HMEJ), 
base editing, and recombinase cas9 (recCas9)] have been developed that might make it possible to correct genetic mutations in non-dividing cells [12-17].

\section{Box 1. CRISPR-Cas9}

The CRISPR system is a defensive tool of archaea and bacteria against invading DNA that has been harnessed for genome editing in plants and animals [18]. This CRISPR system provides adaptive immunity in prokaryotes, by degrading foreign DNA and inserting these sequences in CRISPR loci where they are separated by repetitive sequences [19]. Subsequent invasion results in transcription of these sequences, named CRISPR RNA's (crRNAs, or also guide RNA's (gRNAs)), that then guide Cas effector protein to the complementary DNA sequence in the invading organism. Upon binding, the target region is cleaved by a single-protein Cas effector protein (class 2) or large multi- subunit protein complexes (class 1). These two classes of Cas effector proteins are further diversified into 6 types (type I-III (class 1) and type IV-VI (class 2). The invader sequence is discriminated from the native sequence by a short, conserved sequence, named the protospacer adjacent motif (PAM), located on the non- complementary strand.

CRISPR-Cas9, and especially Cas9 from streptococcus pyogenes (SpCas9), is the most extensively studied class 2 system. Cas9, as well as few other Cas effectors, require a second RNA molecule called trans-activation crRNA (tracr-RNA) [20]. During transcription of the crRNA, a long transcript (pre-crRNA) is formed, and repetitive sequences bind to the complementary regions in the tracrRNA. Host cell-derived RNAse III then cleaves these RNA duplexes from the pre-crRNA. tracrRNA and crRNA can be used as single chimeric sequence, named single guide RNA (sgRNA). Subsequent to recognition of the PAM sequence at the $3^{\prime}$ of the target sequence, Cas9 unwinds the target sequence allowing target recognition by the gRNA. This unwinding forms an R- loop that progresses away from the PAM sequence [21]. Cas9 cleaves both DNA strands 3 nucleotides upstream of the PAM sequence, by two distinct domains, namely the HNH and RuvC/RNaseH-like endonuclease domains. Perfect complementation to the target sequence allows for a conformational change in the HNH domain, which then regulates the cleavage of the RuvC domain via allosteric communication, enabling high-fidelity cleavage of both strands [22, 23].

This review discusses these recent strategies for in vivo gene editing in non-dividing cells. We not only describe novel NHEJ-based strategies in a mutation type-oriented viewpoint (Figure 1), but also suggest approaches that would enable the use of these strategies for a wider range of mutations than those described in the initial reports (Box 
3). We also discuss the recent evidence that HDR may not be impossible in non-dividing cells.

We first describe in vivo gene editing strategies requiring a donor template, followed by strategies operative in a donor-independent way (Figure 1). We also briefly discuss the application of such strategies in other setting such as quiescent stem cells, cancer modelling, organ cultures and etc. (Box 4). 
Box 2. HDR-dependent In vivo gene editing in non-dividing cells

The major challenge for the in vivo use of programmable nucleases in non-dividing compared with dividing cells is the low efficiency of HDR [1]. HDR is restricted to the $S$ and $G 2$ phases of the cell cycle, which makes non-dividing cells not amenable to precise gene editing and repair by HDR [1]. Nevertheless, there are a number of circumstance wherein HDR mediated gene editing has been used successfully in non-dividing cells (Table I):

(i) the corrected cells have a significantly increased fitness over non-corrected cells, i.e. even if only very few cells have been genetically corrected, they can re-enter the cell cycle, divide and repopulate the organ (for instance genetically corrected mature hepatocytes in hereditary tyrosinemia type I, copper storage disorder and biliary acid transporter defects $[2,3])$;

(ii) the gene functions in a non-cell autonomous manner, and even correction of a limited number of cells is sufficient to treat the disease (for instance for factor VIII in haemophilia [4]);

(iii) the protein has a cell-autonomous function but can also be taken up from the extracellular milieu; and the levels of protein secreted from a limited number of edited cells is sufficient to reverse the phenotype (for instance in lysosomal storage disorders such as mucopolysaccharidosis (MPS) I and II [5]).

Table I. Clinical trials and preclinical examples of HDR dependent in vivo gene editing in non- dividing cells

\begin{tabular}{||l|l|l|l|l|l|l|}
\hline Disease Type & $\begin{array}{l}\text { Specific } \\
\text { circumstance }\end{array}$ & Target Cell & Nuclease & Approach & $\begin{array}{l}\text { Study } \\
\text { phase }\end{array}$ & $\begin{array}{l}\text { Clinicaltrials.Gov } \\
\text { Identifier/Ref }\end{array}$ \\
\hline Hemophilia B & $\begin{array}{l}\text { Non-cell } \\
\text { autonomous }\end{array}$ & Hepatocyte & ZFN & Knock in & Phase I & NCT02695160 \\
\hline MPS I & $\begin{array}{l}\text { Extracellular } \\
\text { uptake }\end{array}$ & Hepatocyte & ZFN & Knock in & Phase I & NCT02702115 \\
\hline MPS II & $\begin{array}{l}\text { Extracellular } \\
\text { uptake }\end{array}$ & Hepatocyte & ZFN & Knock in & Phase I & NCT03041324 \\
\hline Tyrosinemia & $\begin{array}{l}\text { Increased } \\
\text { fitness }\end{array}$ & Hepatocyte & Cas9 & Correction & Preclinical & [3] \\
\hline
\end{tabular}




\section{Donor dependent strategies for in vivo genome editing in non-dividing cells}

To bypass the problem of the low efficiency of HDR in non-dividing cells, it is possible to exploit other DNA repair pathways for precise in vivo gene editing. Several nonhomologous repair methods require -as for HDR - that a donor template is available, but they can also operate throughout the interphase cell cycle and can, therefore, theoretically be exploited for genome editing in non-dividing cells [11].

\section{Non-homologous end-joining (NHEJ)-based strategies (e.g. ObliGaRe, HITI, Cpf1)}

Although NHEJ is known to be a fallible repair system, describing NHEJ as a mostly 'error-prone' pathway may be an oversimplification as there is significant evidence that NHEJ can operate accurately and, hence, contribute to precise gene repair in nondividing cells [24]. Here we describe three NHEJ based methods based on blunt (HITI) or sticky (ObLiGaRe and Cpf1) DSB ends generated by nucleases (Figure 2).

Obligate ligation-gated recombination (ObLiGaRe) is a knock-in NHEJ based strategy devised by Maresca and colleagues [25]. ObLiGaRe is based on obligate heterodimeric ZFNs or TALENs to form a Fokl heterodimer that then cleaves the target sequence. The ObLiGaRe system is based on ligation of a donor plasmid into a DSB site by designing a ZFN (or TALEN) target site in the donor plasmid identical to the one in the genome. The ZFN site in the donor is in the reverse orientation to preclude reconstruction of the ZFN target site (Figure $2 \mathrm{a}$ and $2 \mathrm{~b}$ ). Using this system in dividing cells, a precise in vitro knockin of a $15 \mathrm{~kb}$ insert sequence was achieved in 3 of 4 clones, but only after antibioticbased selection. As ObLiGaRe is based on NHEJ, it could be employed in non-dividing cells, although this still needs to be proven. In addition, in vivo testing has not yet been done. Although ObLiGaRe results in precise gene editing, there will be an addition of half a ZFN target site in the genome, precluding fully precise gene repair.

Homology-independent targeted integration (HITI), devised by Suzuki and colleagues [12] and broadly tested in vitro and in vivo, is another knock in NHEJ-based strategy very similar to the ObLiGaRe-based method. The difference between the two methods is the type of nuclease used: while HITI uses the CRISPR system, ObLiGaRe is based on obligate 
heterodimeric ZFNs or TALENs. For HITI, the donor sequence is flanked by one or two (for the circular or linear donors respectively) sgRNA target sites (Figure 2a and 2c). There is a third identical sequence, but in the reverse orientation, in the target locus wherein the donor DNA is pasted. This approach supports knock-in of a sequence, mostly in the correct orientation, as an intact sgRNA target sequence persists when recombination occurs in the reverse orientation. HITI permits knock-in in both dividing and non-dividing cells. Using the HITI strategy in primary neurons, $\pm 90 \%$ of the targeted cells showed precise insertion of the HITI donor. HITI was also tested to knock-in an entire exon in the Royal College of Surgeons rats, a well-established model for retinitis pigmentosa. Analysis of the DNA sequence from the targeted eyes showed precise integration of the $\mathrm{HITI}$ donor with a frequency of $\pm 30 \%$ at $3^{\prime}$ junctions and $95 \%$ at $5^{\prime}$ junctions. Different frequencies of indels were also observed following HITI-based gene editing in other organs, including brain, liver, heart and muscle. Thus, HITI can result in NHEJ-based precise gene editing, but does not exclude non-precise editing outcomes.

Cas12a (former Cpf1) is a class II type V endonuclease causing staggered cuts, creating a 5 bp long $5^{\prime}$ overhangs $[16,26]$. The produced sticky ends created by Cas12a/Cpf1 are particularly advantageous for NHEJ based gene editing, allowing insertion of a donor in the correct orientation in dividing and non-dividing cells. Aside from making sticky ends, there are other features of Cas12a/Cpf1 that make this enzyme an interesting choice, especially for in vivo applications. These include (i) pre-crRNA self-processing (simplifying multiplex genome editing); (ii) no need for a tracRNA by which Cas12a/Cpf1 is 140 amino acids smaller than Cas9; (iii) a T rich PAM recognition site allowing application in AT rich sequences; (iv) and having little to no off-target effects in human cells $[16,27,28]$. Although it is theoretically possible to deploy the staggered ends made by Cpf1 for NHEJ-mediated precise gene repair in non-dividing cells, this has not yet been experimentally demonstrated. The Cas12b (former $\mathrm{C} 2 \mathrm{c} 1$ ) is another type $\mathrm{V}$ system making staggered cut sites, resulting in a 7 base-pair overhang, that could be used for sticky end NHEJ-mediated gene editing [26, 29].

\section{Microhomology-mediated end-joining (MMEJ)- based strategy}


MMEJ is an alternative NHEJ repair pathway independent of $\mathrm{Ku}$, that uses microhomologous sequences of 5-25 nucleotides, flanking the DSB, to join the broken ends [30]. While NHEJ preferentially operates in G1, MMEJ occurs predominantly in the G2 and S phases. Although limited end resection with slower kinetics is possible in G1 cells, this might be sufficient is for most MMEJ processes. Therefore, this pathway could potentially be harnessed to perform gene editing in non-dividing cells. The precise integration into target chromosome (PITCH) strategy, described by Nakade and colleagues, is based on the MMEJ pathway [31]. PITCH employs either TALENs or CRISPRs. CRISPR-mediated PITCH (CRIS-PITCH) is based on the incorporation of two different sgRNA target sites flanking the sequence to be knocked-in (Figure $2 \mathrm{~d}$ and $2 \mathrm{e}$ ), and a third sgRNA site in the target locus. The three different sgRNA are designed such that they will produce micro-homologous regions following DSB. This micro-homology determines the correct orientation of ligation. Using the PITCH approach, a targeted knock-in was performed in silk worm and frog embryos, as well as primary neurons. Although precise knock-in was shown in targeted cells, unwanted non-precise outcomes bearing insertions and deletions were also observed [12, 31].

\section{Homology-mediated end joining (HMEJ)-based strategy}

HMEJ uses a donor construct with \pm 800 bp homology arms flanked by sgRNA sites. To create a knock-in, the donor construct will be cut out from the backbone inside the cell by co-delivered sgRNAs. HMJE allowed precise in vivo targeted knock-in in adult mouse brain and liver with efficiencies greater than either MMEJ, NHEJ or HDR. It was shown that the repair pathway involved in HMEJ-based editing of non-dividing cells, although homology dependent, is different from HDR [13]. However, further investigations will be needed to elucidate the molecular mechanism involved in HMEJ-based DNA repair.

\section{RecCas9-based strategy}

RecCas9 was generated by Chaikind and colleagues, by fusing dead Cas9 (dCas9) to a serine recombinase catalytic domain [17]. The RecCas9 fused protein could be used as a donor-dependent strategy to create insertions, or as a donor-independent method to 
cause deletions, inversions or chromosomal translocations, based on the substrate orientation (Box 3). Using RecCas9, Chaikand and colleagues demonstrated that a seamless deletion of $14 \mathrm{~kb}$ size could be created in a genomic sequence of human cells. However, an attempt to create an insertion failed, and whether or not RecCas9 can catalyse insertions is still to be determined. RecCas9 recognizes endogenous target sites in the genome. However, there are only 450 target sites for RecCas9 in the genome. Replacing Gin recombinase with other recombinases and/or using other types of CRISPR systems with different PAM sequences, might expand the target sites, and overcome this limitation. Because RecCas9 mediates recombination-independent DNA repair, it might be more efficient than all other programmable nucleases in non-dividing cells.

\section{Transcription coupled (TC) DSB repair (TC-DSB)-based strategy}

Even if non-dividing cells do not transfer genetic information to other progenies, their long-term genome stability is crucial. Therefore, DSBs in actively transcribed genes need to be repaired with sufficient accuracy [32]. One such mechanism might be DNA repair coupled with transcription termed transcription coupled NER (TC-NER), which has been suggested long ago [32-35]. It has also been reported that RNA, rather than DNA, can be used as a template for DNA repair at transcriptionally active sites in both yeast and human cells $[33,34,36-41]$. Moreover, there is evidence that the HDR pathway can use an RNA transcript-template to edit DNA in active transcriptional sites damaged by DSB of human cells in the G0/G1 phase. These studies challenge the view that HDR occurs only in the $\mathrm{G} 2$ or $\mathrm{S}$ phase and the traditional view that HDR requires DNA as a template $[11,39]$. Concordantly a recent study demonstrated that HDR in non-dividing cardiomyocytes does not depend on S-phase entry [42]. Using adeno-associated virus (AAV) to carry the HDR donor, efficient precise correction was achieved in cardiomyocytes in vivo in mice. Another study suggested that NHEJ but not HDR was involved in error-free repair of DSBs in active genes of human cells, using the nascent RNA as a template [40]. A strong association between NHEJ factors and transcription was observed, with increased levels of NHEJ when transcription was induced [40]. Another report demonstrated that homologous chromosomes in human cells in in 
G0/G1 initiate contact at DSB sites in transcribed regions [43], while yet another study, using genome wide profiling, described accumulation of HDR proteins at active transcription sites [36]. Thus, even if mechanisms underlying RNA templated DNA repair in non-dividing cells needs further elucidation, this concept has a direct application with regard to precise gene repair in non-dividing cells.

\section{Donor-dependent strategies: possible approaches and pitfalls}

ObLiGaRe, HITI, PITCH and HMEJ can be used to reverse deletion mutations resulting in exon skipping, or whole exon deletions, by inserting a complete exon (insertion approach). These strategies can also be used to correct mutations by knocking in the cDNA of a full gene or part of a gene (downstream of the mutation), maintaining RNA transcription under the control of the native promoter (Figure 1). In addition, ObLiGaRe, HITI, PITCH and HMEJ can also be used to replace a mutant by a wild-type sequence (replacement approach) (Box 3). RecCas9 could theoretically also be applied to reverse such mutations by inserting new sequences, although RecCas9 mediated deletion is much more efficient than insertion. Cas12a, on the other hand, may not be suitable for correction of a deletion, as reconstruction of the cut-site following donor integration cannot be excluded (Box 3).

Donor-dependent strategies are different (i) in the precision of the design and the possibility of predicted unwanted outcomes, (ii) the efficiency of the strategy in nondividing cells, (iii) and the frequency of additional mutations.

(i) While HITI and ObLiGaRe result in the addition of half a ZFN/sgRNA target site in the genome, HMEJ, PITCH and RecCas9 do not pose this design limitation. However, although PITCH and HMEJ strategies use microhomologous and homologous sequences to enable the correct direction of the insert integration, they do not preclude unwanted editing outcomes such as NHEJ mediated ligation of the insert in both correct and reverse orientations, irrespective of homologies [12]. This unwanted outcome is more likely for the PITCH than the HMEJ approach. The disadvantage of the HITI and ObLiGaRe strategies, namely the incorporation of half a ZFN/sgRNA target site in the 
genome, has, however, the benefit that insertion in the reverse orientation is disfavoured.

(ii) The efficiency of different methods has only been compared in a few studies. Suzuki and colleagues demonstrated that the knock-in efficiency by the HITI-approach is higher compared to PITCH and HDR in both dividing and non-dividing cells in vitro, and more efficient than HDR in non-dividing cells in vivo [12]. Yao and colleagues found that the efficiency of HMEJ was higher than NHEJ, PITCH and HDR, as they demonstrated that HMEJ much more efficiently corrected mutated genes in adult brain and liver than either MMEJ, NHEJ (simple NHEJ not by HITI), or HDR in liver [13]. Comparisons between RecCas9 or ObLiGaRe approaches and other approaches have not yet been published. In any case, more studies are needed to further define the efficiency of performing all donor-dependent strategies.

(iii) The occurrence of indels has been observed in all NEHJ/MMEJ/HMEJ approaches, with variable frequencies between different strategies and different tissues [12, 13]. Therefore, designing the ZFN/sgRNA target sequences in less sensitive intronic regions might prevent undesired consequences. Indels did not occur using RecCas9 for deletion [17]. 


\section{Donor independent strategies for in vivo genome editing in non-dividing cells}

Donor independent strategies do not require HDR, therefore they should be possible in non-dividing cells. Any type of programmable nuclease can potentially be used independent of a donor to perform NHEJ based gene editing. Donor independent strategies include Base-editing- or RecCas9-mediated gene editing.

\section{Base editing strategy}

Base editing was originally described by Komor and colleagues, by engineering the base editor (BE) (for a review on base editing systems see [44]). BE is an engineered Cas9 cytidine deaminase fusion enzyme that creates a $C$ to $T$ (or $G$ to $A$ ) conversion within positions -16 to -12 from the PAM sequence [15]. Since the initial description, BE has been extensively improved and expanded. The initial BE (BE1) fused a dCas9 to Cytidine deaminase from rat ( $\mathrm{APOBBEC1)}$. Although BE1 showed efficient conversion of $\mathrm{C}$ to $\mathrm{T}$ in cell-free experiments, a much lower efficiency was observed in cells, because cells repair U:G mismatches causing $U$ excision by uracil DNA glycosylase (UDG) as a part of the base Excision Repair (BER) pathway, and reversion of the $U->G$ to $C->G$. To overcome this problem a second BE (BE2) was generated wherein an UDG inhibitor (UGI) was added to BE1, increasing the efficiency by three-fold.In the third iteration (BE3), dCas9 was replaced by a Cas9-nickase-D10A which nicks the non-edited strand (G), as the mismatch repair (MMR) pathway prefers to correct the base on the nicked strand. Therefore, the mismatched U-G is replaced by U-A and the desired T-A is formed upon DNA repair or replication. BE3 was 6-fold more efficient than BE2. BE3 was further upgraded to BE4, by adding two copies of UGI, optimizing the linker, and adding a GAM protein [45]. GAM proteins (from Mu bacteriophage) bind and protect DSBs that can occur because of two nicks, which in the BE4 strategy can be caused by BER combined with the nick caused by Cas9-nickase-D10A on the opposite strand. YEE-BE3 is another iteration of BE3 which has an activity window of 1-2 bases precluding the unwanted C to T conversion, although this conversion always results in silent mutations if this occurs in the third position of a codon [46]. BEs have been delivered in vivo to the murine inner 
ear (by lipid nanoparticle) and liver (adenoviral vector), yielding gene editing efficiencies of $34 \%$ and $1.5 \%$, respectively $[15,47,48]$. Target-AID is another base editing system which uses a D10A Cas9 nickase and UGI, similar to BE3. This system uses a different cytidine deaminases and protein fusion sequence [49].

\section{RecCas9 strategy}

RecCas9, also described above for donor-dependent approaches, can successfully be used to create a deletion in a donor-independent strategy, to reverse translocation mutations provided that the recombinase recognition sites are present flanking the translocated sequences. Another mutation that might be reversed by RecCas9 is an inversion, provided that suitable substrate does exist flanking the inverted region. Further investigation of additional RecCas9 recognition sites might make these applications possible (Box 3 ).

\section{Donor-independent strategies: possible approaches and pitfalls}

Some types of mutations that can be corrected by donor independent strategies include among others, gain of function (GOF) mutations, inversions, translocations, substitutions, insertions, duplications or mutations that can be reversed by indels, as long as loss of one intact copy of the gene does not cause disease.

Deletions can be created by using a pair of sgRNAs together with Cas9 or other CRISPR systems, two pairs of ZFN or TALEN, leading to repair by NHEJ. One needs to, however, be cognizant that creation of indels, which might consist of multiple bp, could unexpectedly disrupt non-coding RNAs [50-53]. Alternatively, recCas9 could also be used to perform deletions, provided that recognition sequences are present flanking the target site.

One problem with removal of insertions and duplications, is that when two sgRNA and Cas9 are used, it is possible that the sequence between the two sgRNAs can re-integrate in a reverse orientation, which will block the sgRNA recognition sites and lock the insert inside the target site, however we provide suggestions to circumvent this problem (Box 
3). Deletion approaches can target GOF mutations, mutations reversible by exon skipping and intronic cryptic splicing site mutations

Generally, for GOF mutations knocking out the gene is enough to reverse the phenotype as long as hemizigousity does not create a LOF phenotype. The main problem for genome editing of GOF mutations, regardless of the cell type, is that discriminating between the mutant and wild type allele using programmable nucleases especially CRISPR-Cas9 when the mutation is a single base substitution or loss of one or a few bps. However, mismatch at even one (or a few) bp is not tolerated when these are located in the 'sensitive core' (-16 to -13 of the PAM sequence of SpCas9; or between 1 and 18, except 8 and 9 distal to the PAM protospacer positions for Cas12a) [54, 55]. Therefore, allele specific knock out of GOF mutations could be possible if for example sgRNAs are chosen such that the mutation or a polymorphism in the diseased allele falls within this sensitive core. Alternatively, one could exploit the possibility that the mutant allele differs from the wild type in a PAM disturbing nucleotide, either due to the mutation itself or a polymorphism in the gene sequence [56] .

NHEJ induced indels can also be used to reframe a frameshift LOF mutation or to disrupt a frame in GOF mutations. Although the result of reframing will likely not be precise, as a few bases may be deleted, this might be tolerated in some genes [57].

Base editing approaches can be used to target $\mathrm{T}>\mathrm{C}$ substitution mutations in nondividing cells [15]. Other substitutions could be correctable by using different types of deaminases and expanding the substrate of BEs in the future. Base editing approaches could also be used to knock-out GOF mutations by creating a stop codon via substitution of $C$ by $T$ or $G$ by $A$ in glutamine, arginine or tryptophan codons $[47,58]$.

RecCas9 has been used to create deletions, although adaptations will be needed to expand the substrate sequences.

Other donor independent approaches are inversion and translocations. However, the efficiency of such approaches for in vivo gene therapy in non-dividing cells is not clear yet (Box 3). 
Box 3. Further evaluation and / or adaptations required to current methods of genome editing in nondividing cells:

A number of the candidate donor-dependent and -independent gene editing approaches described, lack experimental proof of concept, and / or require further optimisation.

\section{Donor dependent replacement approach}

These are methods to replace a mutant sequence in the target locus with a donor template. RecCas 9 could be used to replace any mutation by catalysing cleavage, strand-exchange and re-ligation of the donor DNA [17]. Cas12a/Cpf1-based NEHJ could in principle be used in a replacement approach, by combining four different sgRNAs. The sgRNAs should then be designed such that compatible sticky ends are created between the insert and the target locus. To lock the insert and prevent subsequent removal of the integrated insert, a synonymous mutation that disrupts the PAM sequence in the insert should be included. Theoretically, this approach would be able to correct any type of mutation.

Extrapolating the CRISPR-mediated precise integration into target chromosome (CRIS-PITCh), homologous-mediated end joining (HMEJ), homology-independent targeted integration (HITI) and Obligate Ligation-Gated Recombination (ObLiGaRe) to a donor-dependent replacement approach, is in principle possible. However, the predicted unwanted outcomes such as, inversions and deletions of mutant sequence in all of these methods, and unwanted NHEJ mediated ligation of the insert, when using PITCh and HMEJ, make these designs not suitable for in vivo applications.

Donor independent inversion vs. deletion approach

Simple cutting of any sequence using a pair of sgRNA and a SpCas9 might result in either deletion or inversion of the excised sequence. However, it is possible to disfavour one of these events by using obligate heterodimeric ZFN and TALEN, or Cas9-Fokl (figure I). Alternatively, RecCas9 can mediate inversions if a substrate sequence is present. Removal of an inverted sequence with a pair of sgRNA has been performed by RecCas9, to reverse a haemophilia mutation in vitro or cancer modelling in vivo, but exploration to an in vivo therapeutic application in preclinical models is still needed [59, 60].

\section{Donor independent translocation approach}

Translocation mutations can be categorized based on the number of DSBs involved into four classes: simple (one), reciprocal (two), transposition (three) and complex (more than three DSBs) translocations. In vivo and in vitro models of reciprocal translocations have been created using recombinases and the CRISPR system [61, 62]. The possibility to use such an approach to reverse translocation mutations for therapeutic purposes will however, require further work. 
Box 4. Additional applications of gene editing strategies in non-dividing cells:

\section{Targeting other cell types}

Strategies suitable for gene editing in non-dividing cells could be used to target stem cells that are known to be quiescent (G0) in the diseased organ, and thus create a reserve of edited stem cells for tissue regeneration. This is especially important in high turnover tissues such as epidermis, where editing the rapidly dividing transient amplifying pool will only temporarily correct mature skin cells, while correcting stem cells would lead to long-term therapeutic effects [63].

As non-HDR methods are also more efficient than HDR, even in dividing cells [12, 15], strategies described for non-HDR mediated gene editing could also be used in dividing cells for both in vivo and ex vivo gene editing.

\section{More than correcting a gene}

In addition to correcting a mutation, in vivo gene editing strategies for non-dividing cells would also allow insertion of a therapeutic gene in a 'safe harbor'. For instance, precise HDR mediated knock in of proteins in hepatocytes downstream of the albumin promoter, may represent a universal platform for the production of secreted proteins (e.g. human factors VIII and IX for hemophilia A and B) or proteins that can be taken up by diseased cells (e.g. lysosomal enzymes for mucopolysaccharidosis) [64, 65]. This platform is especially important because it provides a permanent therapy for a diverse range of genetic and non-genetic disorders. Similarly, non-HDR strategies such as homology-independent targeted integration (HITI), obligate ligation-gated recombination (ObliGaRe), precise integration into target chromosome (PITCh) or homology-mediated end-joining (HMEJ) could be used to insert any sequence of interest in a desired locus in non-dividing cells via an insertion approach (figure).

\section{Gene editing in organ cultures}

Methods for in vivo gene editing in non-dividing cells could also be used to ex vivo gene edit donor explants or organ cultures. Donor explants, that can be maintained in culture, such as cornea, would be a candidate for such manipulations. Alternatively, genome manipulation of non-dividing cells in human brain slice for instance for applying optogenetic tool, could also be achieved by for instance HITI, which is highly efficient in primary neurons $[12,66,67]$.

\section{Modeling disorders based on somatic mutations}

In vivo manipulation of non-dividing cells could be used to model disorders based on somatic mutations in mature non-dividing cells, such as cancer and neurological disorders [68]. Base editor (BE), in particular, could be used to model cancer, as more than $50 \%$ of nonsense mutations across all cancers result in a stop codon, and $>50 \%$ of such stop codons could be created by BE [58]. 


\section{Conclusion and future perspectives}

In vivo gene editing using programmable nucleases including the CRISPR system, constitutes the only approach for curing many genetic disorders. Low efficiency of the HDR repair pathway in non-dividing cells, which is the status of differentiated cells in almost all organs, does, however, limit the use of this technology to only a few conditions. Nevertheless, alternative repair pathways or strategies discussed in this review are being developed to overcome this hurdle, even if some of these strategies have not been fully tested in vivo and in non-dividing cells. Additionally, transcriptioncoupled DSB repair (TC-DSB) repair in non-dividing cells, if proven to be efficient, could be co-opted to artificially enable gene editing in non-dividing cells. We also discuss that type of mutation dictates which editing approach will be more suitable. Finally, we discuss that problems with editing efficiency frequency, and the possibility of unwanted outcomes exist with different of these approaches, and are important factors that should be taken into account when choosing a specific method. There are still more questions and uncertainties which have to be addressed before proceeding to clinic, that have been summarised in 'Outstanding Questions'. 


\section{References}

1. Cox, D.B. et al. (2015) Therapeutic genome editing: prospects and challenges. Nat Med 21 (2), 121-31.

2. Paulk, N.K. et al. (2010) Adeno-associated virus gene repair corrects a mouse model of hereditary tyrosinemia in vivo. Hepatology 51 (4), 1200-8.

3. Yin, H. et al. (2014) Genome editing with Cas9 in adult mice corrects a disease mutation and phenotype. Nat Biotechnol 32 (6), 551-3.

4. Li, H. et al. (2011) In vivo genome editing restores haemostasis in a mouse model of haemophilia. Nature 475 (7355), 217-21.

5. Desnick, R.J. and Schuchman, E.H. (2002) Enzyme replacement and enhancement therapies: lessons from lysosomal disorders. Nat Rev Genet 3 (12), 954-66.

6. Fagiuoli, S. et al. (2013) Monogenic diseases that can be cured by liver transplantation. J Hepatol 59 (3), 595-612.

7. O'Connor, T.P. and Crystal, R.G. (2006) Genetic medicines: treatment strategies for hereditary disorders. Nat Rev Genet 7 (4), 261-76.

8. Kole, R. et al. (2012) RNA therapeutics: beyond RNA interference and antisense oligonucleotides. Nat Rev Drug Discov 11 (2), 125-40.

9. Kimbrel, E.A. and Lanza, R. (2015) Current status of pluripotent stem cells: moving the first therapies to the clinic. Nat Rev Drug Discov 14 (10), 681-92.

10. Gaj, T. et al. (2013) ZFN, TALEN, and CRISPR/Cas-based methods for genome engineering. Trends Biotechnol 31 (7), 397-405.

11. Hustedt, N. and Durocher, D. (2016) The control of DNA repair by the cell cycle. Nat Cell Biol 19 (1), 1-9.

12. Suzuki, K. et al. (2016) In vivo genome editing via CRISPR/Cas9 mediated homologyindependent targeted integration. Nature 540 (7631), 144-149.

13. Yao, X. et al. (2017) Homology-mediated end joining-based targeted integration using CRISPR/Cas9. Cell Res 27 (6), 801-814.

14. Sakuma, T. et al. (2016) MMEJ-assisted gene knock-in using TALENs and CRISPR-Cas9 with the PITCh systems. Nat Protoc 11 (1), 118-33.

15. Komor, A.C. et al. (2016) Programmable editing of a target base in genomic DNA without double-stranded DNA cleavage. Nature 533 (7603), 420-4.

16. Zetsche, B. et al. (2015) Cpf1 Is a Single RNA-Guided Endonuclease of a Class 2 CRISPR-Cas System. Cell 163 (3), 759-71.

17. Chaikind, B. et al. (2016) A programmable Cas9-serine recombinase fusion protein that operates on DNA sequences in mammalian cells. Nucleic Acids Res 44 (20), 975870.

18. Mali, P. et al. (2013) Cas9 as a versatile tool for engineering biology. Nat Methods 10 (10), 957-63. 
19. Barrangou, R. et al. (2007) CRISPR provides acquired resistance against viruses in prokaryotes. Science 315 (5819), 1709-12.

20. Deltcheva, E. et al. (2011) CRISPR RNA maturation by trans-encoded small RNA and host factor RNase III. Nature 471 (7340), 602-7.

21. Szczelkuna, M.D. (2014) Direct observation of R-loop formation by single RNAguided Cas9 and Cascade effector complexes

PNAS 111 (27), 9798-9803.

22. Sternberg, S.H. et al. (2015) Conformational control of DNA target cleavage by CRISPR-Cas9. Nature 527 (7576), 110-3.

23. Dagdas, Y.S. (2017) A conformational checkpoint between DNA binding and cleavage by CRISPR-Cas9. Sci.Adv 3, eaao0027.

24. Betermier, M. et al. (2014) Is non-homologous end-joining really an inherently errorprone process? PLoS Genet 10 (1), e1004086.

25. Maresca, M. et al. (2013) Obligate ligation-gated recombination (ObLiGaRe): customdesigned nuclease-mediated targeted integration through nonhomologous end joining. Genome Res 23 (3), 539-46.

26. Murugan, K. et al. (2017) The Revolution Continues: Newly Discovered Systems Expand the CRISPR-Cas Toolkit. Mol Cell 68 (1), 15-25.

27. Zetsche, B. et al. (2017) Multiplex gene editing by CRISPR-Cpf1 using a single crRNA array. Nat Biotechnol 35 (1), 31-34.

28. Kim, D. et al. (2016) Genome-wide analysis reveals specificities of Cpf1 endonucleases in human cells. Nature Biotechnology 34 (8), 863-868.

29. Rohovie, M.J. et al. (2017) Virus-like particles: Next-generation nanoparticles for targeted therapeutic delivery. Bioengineering \& Translational Medicine 2 (1), 43-57.

30. McVey, M. and Lee, S.E. (2008) MMEJ repair of double-strand breaks (director's cut): deleted sequences and alternative endings. Trends Genet 24 (11), 529-38.

31. Nakade, S. et al. (2014) Microhomology-mediated end-joining-dependent integration of donor DNA in cells and animals using TALENs and CRISPR/Cas9. Nat Commun 5, 5560.

32. Wei, L. et al. (2016) Transcription-coupled homologous recombination after oxidative damage. DNA Repair (Amst) 44, 76-80.

33. Marnef, A. et al. (2017) Transcription-Coupled DNA Double-Strand Break Repair: Active Genes Need Special Care. J Mol Biol 429 (9), 1277-1288.

34. D'Alessandro, G. and d'Adda di Fagagna, F. (2016) Transcription and DNA Damage: Holding Hands or Crossing Swords? J Mol Biol 429 (21), 3215-29.

35. Meers, C. et al. (2016) DNA repair by RNA: Templated, or not templated, that is the question. DNA Repair (Amst) 44, 17-21.

36. Aymard, F. et al. (2014) Transcriptionally active chromatin recruits homologous recombination at DNA double-strand breaks. Nat Struct Mol Biol 21 (4), 366-74. 
37. Keskin, H. et al. (2014) Transcript-RNA-templated DNA recombination and repair. Nature 515 (7527), 436-9.

38. Ohle, C. et al. (2016) Transient RNA-DNA Hybrids Are Required for Efficient DoubleStrand Break Repair. Cell 167 (4), 1001-1013 e7.

39. Wei, L. et al. (2015) DNA damage during the G0/G1 phase triggers RNA-templated, Cockayne syndrome B-dependent homologous recombination. PNAS 112 (27), E3495504.

40. Chakraborty, A. et al. (2016) Classical non-homologous end-joining pathway utilizes nascent RNA for error-free double-strand break repair of transcribed genes. Nature Communications 7, 13049.

41. Hawley, B.R. et al. (2017) The emerging role of RNAs in DNA damage repair. Cell Death Differ 24 (4), 580-587.

42. Ishizu, T. et al. (2017) Targeted Genome Replacement via Homology-directed Repair in Non-dividing Cardiomyocytes. Sci Rep 7 (1), 9363.

43. Gandhia, M. (2012) Homologous chromosomes make contact at the sites of double-strand breaks in genes in somatic G0/G1-phase

human cells. PNAS 109 (24), 9454-59.

44. Hess, G.T. et al. (2017) Methods and Applications of CRISPR-Mediated Base Editing in Eukaryotic Genomes. Mol Cell 68 (1), 26-43.

45. Komor, A.C. (2017) Improved base excision repair inhibition and bacteriophage $\mathrm{Mu}$ Gam protein yields C:G-to-T:A base editors with higher efficiency and product purity. Sci Adv 3 (8), eaao4774.

46. Kim, Y.B. et al. (2017) Increasing the genome-targeting scope and precision of base editing with engineered Cas9-cytidine deaminase fusions. Nat Biotechnol 35 (4), 371376.

47. Chadwick, A.C. (2017) In Vivo Base Editing of PCSK9 (Proprotein Convertase Subtilisin/Kexin Type 9) as a Therapeutic Alternative to Genome Editing. Arterioscler Thromb Vasc Biol 37, 1741-1747.

48. Rees, H.A. et al. (2017) Improving the DNA specificity and applicability of base editing through protein engineering and protein delivery. Nat Commun 8, 15790. 49. Nishida, K. (2016) Targeted nucleotide editing using hybrid prokaryotic and vertebrate adaptive immune systems. 353 (6305), aaf8729.

50. Ruan, G.X. et al. (2017) CRISPR/Cas9-Mediated Genome Editing as a Therapeutic Approach for Leber Congenital Amaurosis 10. Mol Ther 25 (2), 331-341.

51. Tabebordbar, M. et al. (2016) In vivo gene editing in dystrophic mouse muscle and muscle stem cells. Science 351 (6271), 407-11.

52. Nelson, C.E. et al. (2015) In vivo genome editing improves muscle function in a mouse model of Duchenne muscular dystrophy. Science 351 ( 6271), 403-7. 
53. Xu, L. et al. (2015) CRISPR-mediated genome editing restores dystrophin expression and function in mdx mice. Mol Ther 24 (3), 564-69.

54. Zheng, T. et al. (2017) Profiling single-guide RNA specificity reveals a mismatch sensitive core sequence. Sci Rep 7, 40638.

55. Kleinstiver, B.P. et al. (2016) Genome-wide specificities of CRISPR-Cas Cpf1 nucleases in human cells. Nat Biotechnol 34 (8), 869-74.

56. Bakondi, B. et al. (2016) In Vivo CRISPR/Cas9 Gene Editing Corrects Retinal Dystrophy in the S334ter-3 Rat Model of Autosomal Dominant Retinitis Pigmentosa. Mol Ther 24 (3), 556-63.

57. Zhang, Y. et al. (2017) CRISPR-Cpf1 correction of muscular dystrophy mutations in human cardiomyocytes and mice. Sci Adv 3 (4), e1602814.

58. Billon, P. et al. (2017) CRISPR-Mediated Base Editing Enables Efficient Disruption of Eukaryotic Genes through Induction of STOP Codons. Mol Cell 67 (6), 1068-1079 e4.

59. Park, C.Y. et al. (2015) Functional Correction of Large Factor VIII Gene Chromosomal Inversions in Hemophilia A Patient-Derived iPSCs Using CRISPR-Cas9. Cell Stem Cell 17 (2), 213-20.

60. Maddalo, D. et al. (2014) In vivo engineering of oncogenic chromosomal rearrangements with the CRISPR/Cas9 system. Nature 516 (7531), 423-7.

61. Tanaka, M. et al. (2015) Somatic chromosomal translocation between Ewsr1 and Fli1 loci leads to dilated cardiomyopathy in a mouse model. Sci Rep 5, 7826.

62. Torres, R. et al. (2014) Engineering human tumour-associated chromosomal translocations with the RNA-guided CRISPR-Cas9 system. Nat Commun 5, 3964.

63. Wu, W. (2017) Efficient in vivo gene editing using ribonucleoproteins in skin stem cells of recessive dystrophic epidermolysis bullosa mouse model. PNAS 114 (7), 16601665.

64. Sharma, R. et al. (2015) In vivo genome editing of the albumin locus as a platform for protein replacement therapy. Blood 126 (15), 1777-84.

65. Cornu, T.I. et al. (2017) Refining strategies to translate genome editing to the clinic. Nat Med 23 (4), 415-423.

66. Humpel, C. (2015) Organotypic brain slice cultures: A review. Neuroscience 305, 8698.

67. Andersson, M. et al. (2016) Optogenetic control of human neurons in organotypic brain cultures. Sci Rep 6, 24818.

68. Poduri, A. et al. (2013) Somatic mutation, genomic variation, and neurological disease. Science 341 (6141), 1237758. 


\section{Legends:}

Figure 1. Candidate gene editing approaches for non-dividing cells in vivo.

( $a$ and b) Donor-independent approaches, (c and d) donor-dependent approaches. Boxes with dash outlines are potential approaches which insufficient experimental proof that they can edit genes in vivo in non-dividing cells exists ( $b$ and $d$ ). In each box approaches (in bold letters) and strategies are listed in the left column, targetable mutations for each approach are listed in the middle column, and schematic representation of each approach is shown in the right column.

(right column): an exon in pink color, and a red star indicate the mutated exon; dashed lines show splicing pattern; green triangles represent the manipulation site; Stop: stop codon; PA: poly A sequence.

(box 'a'): the cryptic exon (crypt ex) is shown as an example.

(box ' $c$ '): pink exon2 with a jagged side represent a deletion.

(abbreviations used) BE: base editor, BE 3, BE4 and YEE BE3 are other BE developed from BE3, HITI: Homology-independent targeted Integration, HMEJ: Homologymediated end Joining, ObLiGaRe: Obligate Ligation-Gated Recombination, PITCH: Precise integration into target Chromosome, recCas9: recombinase Cas9, Target-AID: a base editing system very similar to BE3 using a different cytidine deaminase.

\section{Figure 2. The principal of ObLiGaRe, HITI and CRIS-PITCH gene editing strategies}

(a) Homology-independent targeted Integration (HITI)/ Obligate Ligation-Gated Recombination (ObLiGaRe) strategies for targeted knock in (insertion approach) (d) CRIS-PITCH. As an example of a target mutation, exon 2 deletion is shown as a pink exon with a jagged side. A pair of pentagons represent a arget site for sgRNA/ZFN/TALEN. A black scissor indicates cleavage and a grey scissor with a red cross indicate that no cleavage occurs and the insert is locked.

(b) Mechanism of ObLiGaRe strategy, wherein Heterodimeric obligated ZFN are used to ensure the correct orientation of inset ligation. The ZFNs/TALENs make a DSB cut only if two arms on the opposite strands make heterodimers, which is the case for the target 
locus but not homodimers (targeted locus) through a modified Fokl nuclease domain preventing cleavage of the targeted locus.

(c) Mechanism of HITI strategy, wherein the insert is locked in the correct orientation using CRISPR/Cas9. Integration of the insert with correct orientation creates palindromes of a part of sgRNA site (two blue/two pink pentagons) that prevent recleavage of the insert

(e) Mechanism of the CRIS-PITCH strategy demonstrating how microhomologous sequences on the donor and target sequence are formed by CRISPR-Cas9. The sgRNAs target sites are not resent in the targeted locus preventing Cas9 recleavage. (abbreviations used)

Box 1 Figure I. CRISPR/Cas9 system. Cas9 and sgRNA targeting a sequence protospacer, provided that a 3' PAM sequence is present (NGG). The cutting site is shown with black scissor and located 3 bases upstream from the PAM sequence.

Box 3 Figure I. Potential strategies to favour deletion or inversion of a target sequence. The strategies are based on obligated heterodimeric ZFN pairs that can be replaced by TALEN or Cas9-Fokl. Pentagon pairs are ZFN target site. The variant Fokl cleavage domain is depicted in a drop or three-quarter circle.

(a) When there are identical variant Fokl cleavage domains fuse to the ZFN on the sequence that has to be excised from target locus (pink and green ZFNs), deletion is the stable outcome. This is because the inverted sequence reconstructs the ZFN heterodimers (green-blue \& pink-yellow pairs).

(b) When the variant Fokl cleavage domain on the sequence that has to be excised from target locus are different, inversion is the desired outcome. This is because the deletion outcome results in reconstruction of the ZFN heterodimer (Blue-yellow pair). (abbreviations used) 


\section{Outstanding Questions Box}

1. Is the risk inherent to strategies that are mainly based on DSB repair pathways (except from recCas 9 and base editor) for translocations, and hence tumorigenesis?

2. What are the differences in DNA repair pathways used between quiescent, e.g. stem cells that are quiescent but can undergo replication, and terminally differentiated post-mitotic cells such as neurons (but e.g. not hepatocytes), that in theory cannot re-enter cell cycle? Or even between different single cells of a given tissue? How does this affect the efficiency of gene editing?

3. Is RNA-templated DNA repair an alternative for what is considered faithful DNA repair, namely $\mathrm{HDR}$, in non- dividing cells? Is it possible to co-opt this approach to perform precise gene editing in these cells?

4. Does the variability in inserts and deletions (INDEL) frequencies in different organs seen with the Homology-Independent Targeted Integration (HITI) approach also occur when other approaches are used; and what lays at the basis of this?

5. Is it possible to exploit Cas12a as an approach to target a wide range of mutations? Do the complementary sticky ends created by Cas12a allow accurate NHEJ-based gene repair?

6. Is it possible to in vivo correct inversions or translocations with therapeutically acceptable efficiencies? 
a

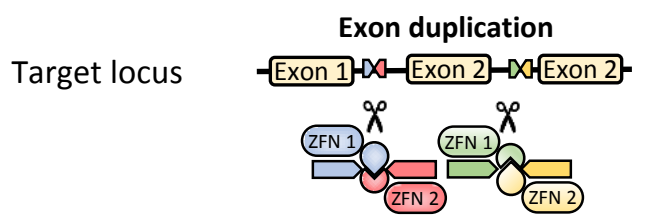

Desired

stable

product

Unwanted

unstable

product

Exon 1-XX-Exon 2-

ZFN 12

Inversion

Exon 1-XX-2 Uox b

Exon inversion

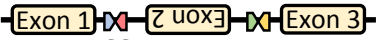

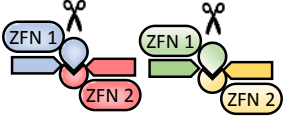

Inversion

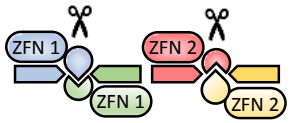

Exon 13X-Exon 2-XX-Exon 3-

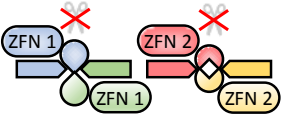

Deletion

Exon 11X-Exon 3-

(2EN1) ${ }^{2}$ 


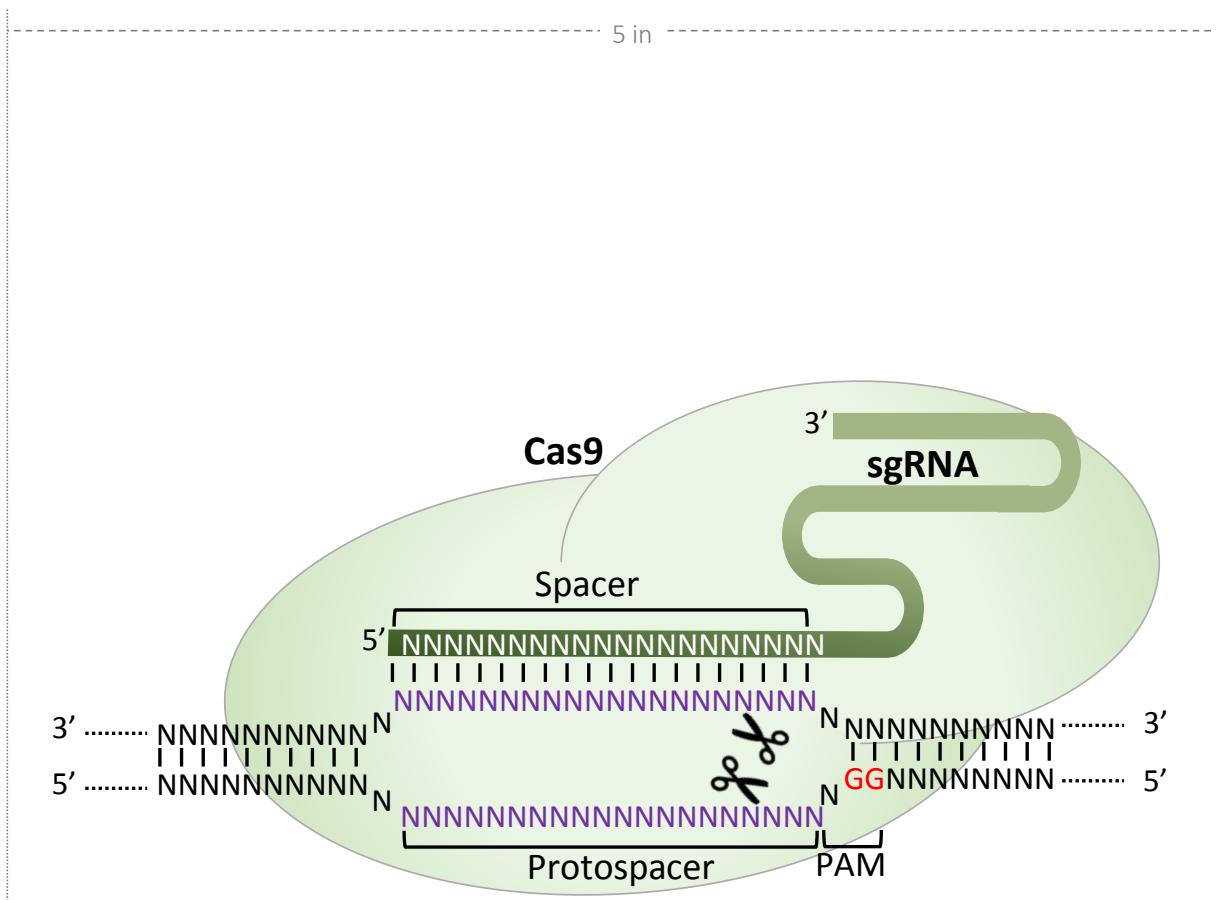




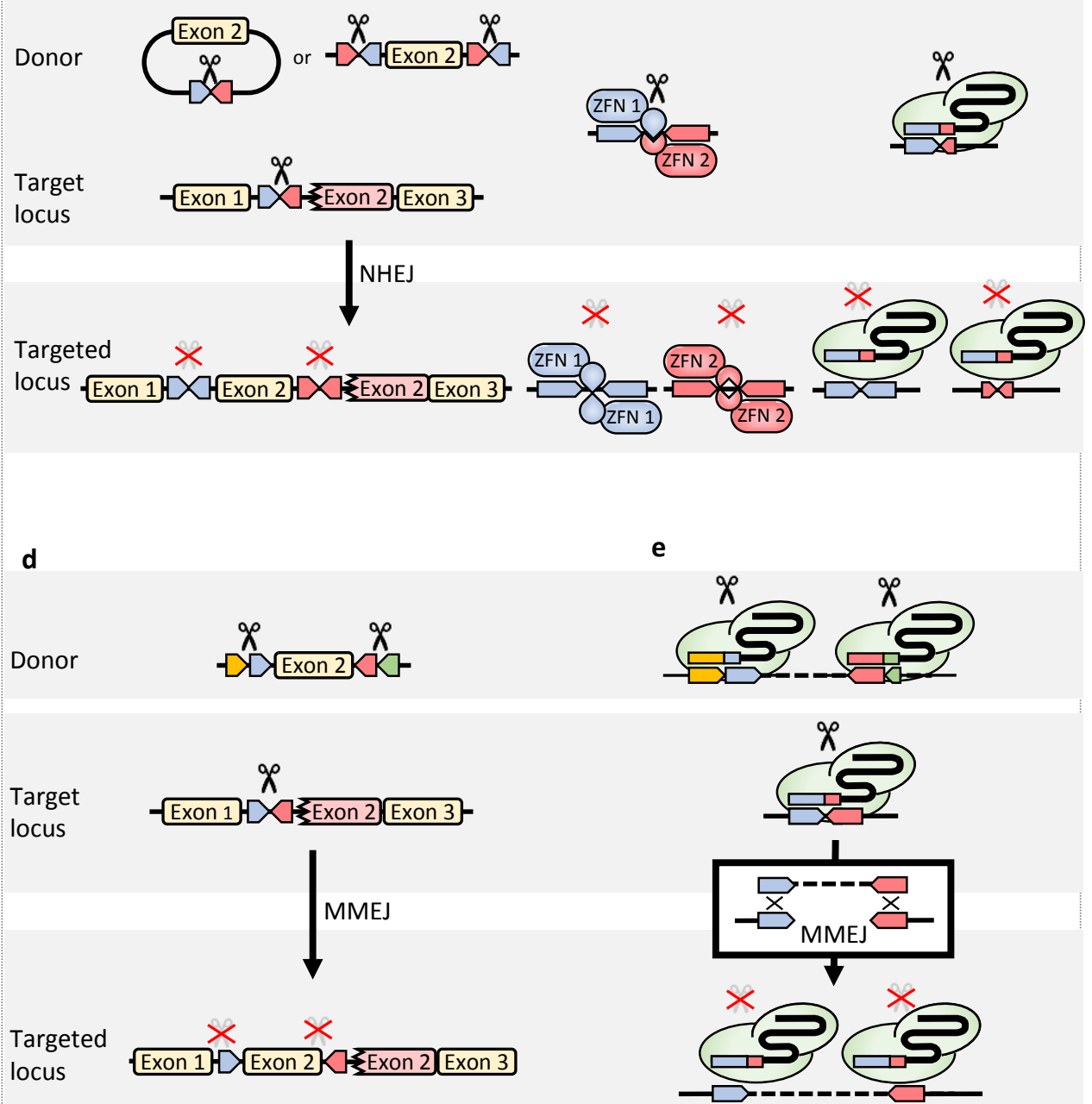

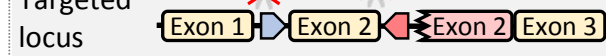


a

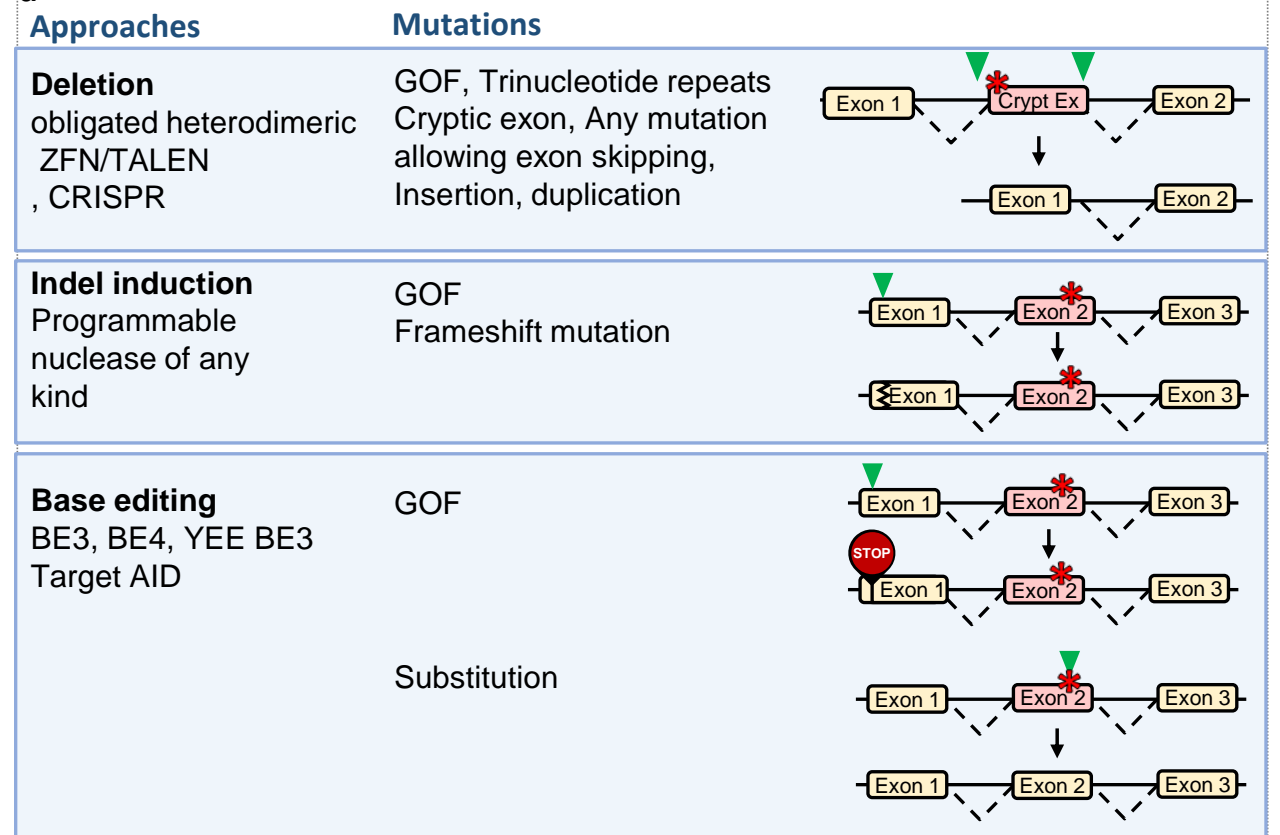

b

Inversion
obligated
heterodimeric ZFN,
TALEN or CRISPR

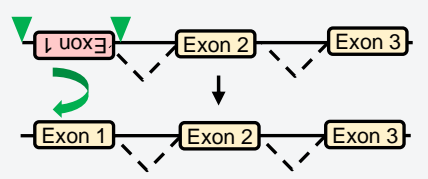

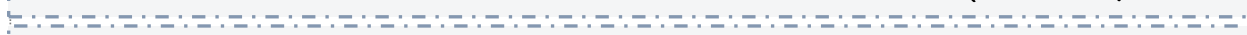

Translocation

recCas9

spCas9

Translocation

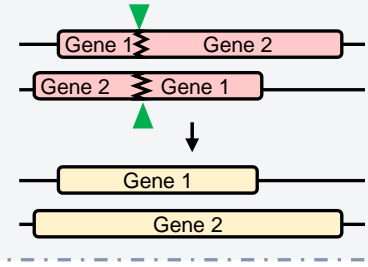

C

Insertion approach

ObliGaRe

HITI

PITCH

HMEJ

RecCas9

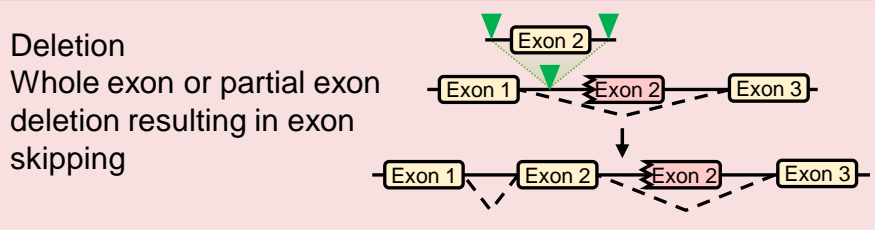

Any type

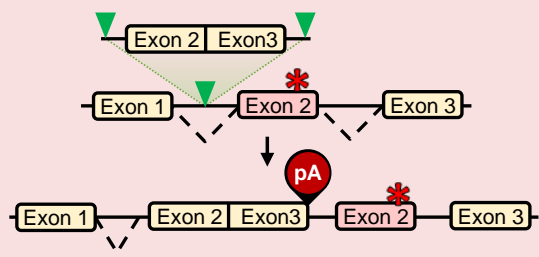

d

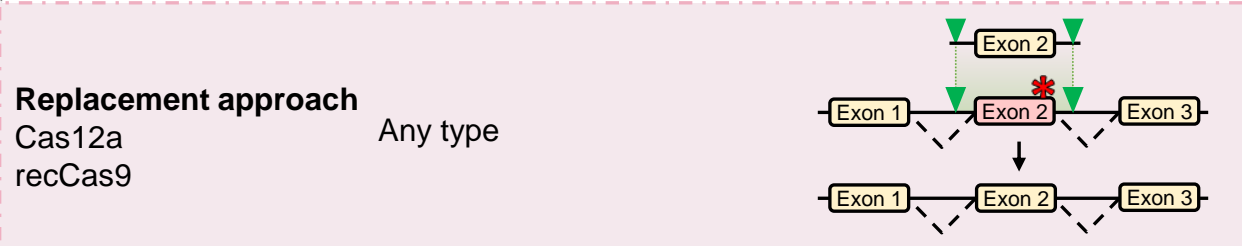

